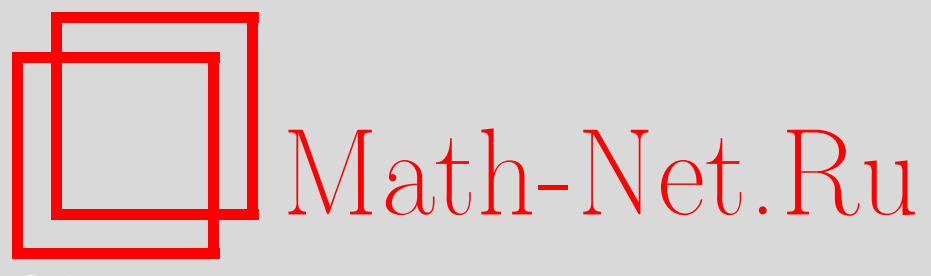

И. Р. Каюмов, О голоморфных движениях $n$-симметричных функций, Матем. заметки, 2010, том 87, выпуск 6, 848-854

DOI: https://doi.org/10.4213/mzm7700

Использование Общероссийского математического портала Math-Net.Ru подразумевает, что вы прочитали и согласны с пользовательским соглашением http://www . mathnet.ru/rus/agreement

Параметры загрузки:

IP: 54.162 .27 .143

26 апреля 2023 г., 11:31:01

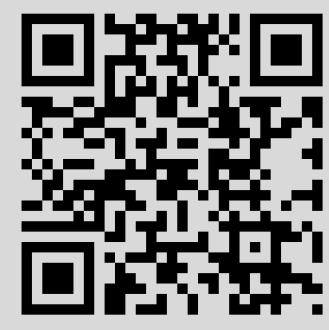


Том 87 выпуск 6 июнь 2010

УДК 517.54

\section{О голоморфных движениях $n$-симметричных функций}

\section{И. Р. Каюмов}

В данной работе мы обобщаем одну задачу, рассмотренную П. Дюреном, об однолистности одного семейства $n$-симметричных функций, порожденных интегралами от функций вида $\exp \left(\lambda \zeta^{n}\right)$. Наш подход основан на использовании обратного преобразования Фабера, критерия однолистности Мартио-Сарваса и $\lambda$-леммы Мане, Сада и Сулливана. В работе также сформулирована гипотеза об однолистности некоторого семейства $n$-симметричных функций, которая является ослабленной формой гипотезы Даникаса-Рушевея об однолистности одного интегрального преобразования голоморфных функций.

Библиография: 10 названий.

1. Введение. Предположим, что функция $f$ голоморфна в единичном круге $\mathbb{D}=\{z:|z|<1\}$. Беккер в работе [1] показал, что если для любого $z \in \mathbb{D}$

$$
\left|\frac{f^{\prime \prime}(z)}{f^{\prime}(z)}\right| \leqslant \frac{1}{1-|z|^{2}},
$$

то функция $f$ однолистна в $\mathbb{D}$. Под однолистностъю отображения понимается инъективность.

Дюрен [2; с. 274], используя этот критерий однолистности, показал, что при $|\lambda| \leqslant$ e/2 функции

$$
f_{n}(z)=\int_{0}^{z} e^{\lambda t^{n}} d t
$$

однолистны в $\mathbb{D}$. В связи с этим возникает естественный вопрос: при каких $\lambda$ функция (1) однолистна в $\mathbb{D}$ ? При $n \leqslant 10$ данная задача была численно решена Гайдуком в [3].

Основной целью данной работы является исследование однолистности более общего класса $n$-симметричных голоморфных в единичном круге функций при больших значениях $n$.

2. Основные результаты. Предположим, что $\Phi(z)$ голоморфна в $\mathbb{D}$ и $\Phi(0)=1$. Определим последовательность $n$-симметричных функций

$$
f_{n}(z)=\int_{0}^{z} \Phi\left(t^{n}\right) d t .
$$

Работа выполнена при поддержке Российского фонда фундаментальных исследований (гранты №№ 08-01-00381, 09-01-12188-офи-м) и Федерального агентства по образованию (госконтракт № П944).

(C) И. Р. КАюмов, 2010 
Напомним, что голоморфная в $\mathbb{D}$ функция $g$ называется $n$-симметричной, если $g\left(e^{i 2 \pi / n} z\right)=e^{i 2 \pi / n} g(z)$ для всех $z \in \mathbb{D}$.

Нетрудно показать, что любая $n$-симметричная функция может быть представлена в виде (2). Например, функции (1) являются функциями вида (2) при $\Phi(z)=e^{\lambda z}$.

Основная проблема нашей работы может быть сформулирована так: для каких функций $\Phi$ найдется натуральное $N$ такое, что функции $(2)$ однолистны в $\mathbb{D}$ для всех $n \geqslant N$ ?

Оказывается, что определяющую роль играет функция

$$
\varphi(z)=z \exp \int_{0}^{z} \frac{\Phi(t)-1}{t} d t
$$

а именно, справедлива

Теорема 1. а) Если существует натуральное $N$ maкое, что для всех $n \geqslant N$ функции $f_{n}$ однолистны в $\mathbb{D}$, то $\varphi$ также однолистна в $\mathbb{D}$.

b) Обратно, если ч отображает круг $\mathbb{D}$ на односвязную область с квазиконформной границей, то найдется натуральное $N$ такое, что функции $f_{n}$ однолистнь в $\mathbb{D}$ при любом $n \geqslant N$.

ДокАзАтельство. Для доказательства а) определим последовательность голоморфных функций $\varphi_{n}(z)=f_{n}(\sqrt[n]{z})^{n}$, другими словами рассмотрим обратное преобразование Фабера однолистных $n$-симметричных функций $f_{n}$. В [4; с. 55] доказывается, что преобразование Фабера переводит однолистные функции в однолистные. Аналогичным образом, нетрудно показать, что обратное преобразование Фабера также сохраняет однолистность. Следовательно, функции $\varphi_{n}(z)$ будут однолистны в единичном круге.

Пусть $\Phi(z)=1+\sum_{k=1}^{\infty} a_{k} z^{k}$. Тогда

$$
\varphi_{n}(z)=z\left(1+\sum_{k=1}^{\infty} \frac{a_{k}}{n k+1} z^{k}\right)^{n} \rightarrow z \exp \sum_{k=1}^{\infty} \frac{a_{k}}{k} z^{k}=\varphi(z) \quad \text { при } \quad n \rightarrow \infty .
$$

Несложно показать, что $\varphi_{n} \rightarrow \varphi$ локально равномерно в $\mathbb{D}$ (ниже мы убедимся, что в квазиконформном случае имеет место равномерная сходимость в $\overline{\mathbb{D}})$. Поскольку функции $\varphi_{n}$ однолистны при больших $n$, то в силу теоремы Гурвица $\varphi$ также однолистна в $\mathbb{D}$.

Для доказательства b) нам понадобится один результат Мартио и Сарваса [5], являющийся следствием результатов, полученных Альфорсом и Берсом. Они доказали, что если $\Omega$ - односвязная область с квазиконформной границей, то существует $\varepsilon>0$ такое, что условие

$$
\left|\frac{\psi^{\prime \prime}(z)}{\psi^{\prime}(z)}\right| \leqslant \varepsilon \rho(z, \Omega)
$$

гарантирует однолистность голоморфной в $\Omega$ функции $\psi$. Здесь $\rho(z, \Omega)-$ плотность гиперболической метрики в $\Omega$.

Из цепочки равенств

$$
\varphi_{n}^{\prime}(z)=f_{n}^{\prime}(\sqrt[n]{z}) z^{1 / n-1} f_{n}(\sqrt[n]{z})^{n-1}=\Phi(z)\left(\frac{\varphi_{n}(z)}{z}\right)^{1-1 / n}=\frac{z \varphi^{\prime}(z)}{\varphi(z)}\left(\frac{\varphi_{n}(z)}{z}\right)^{1-1 / n}
$$


следует, что

$$
\left|\ln \varphi_{n}^{\prime}-\ln \varphi^{\prime}\right|=\left|\ln \frac{\varphi_{n}}{z}-\ln \frac{\varphi}{z}-\frac{1}{n} \ln \frac{\varphi_{n}}{z}\right| \leqslant\left|\ln \frac{\varphi_{n}}{z}-\ln \frac{\varphi}{z}\right|+\frac{1}{n}\left|\ln \frac{\varphi_{n}}{z}\right| .
$$

Мы хотим показать, что

$$
\left|\ln \varphi_{n}^{\prime}-\ln \varphi^{\prime}\right| \rightarrow 0 \quad \text { равномерно в } \overline{\mathbb{D}} \quad \text { при } n \rightarrow \infty \text {. }
$$

С этой целью докажем, что

$$
\left|\ln \frac{\varphi_{n}}{z}-\ln \frac{\varphi}{z}\right| \rightarrow 0 \quad \text { равномерно в } \overline{\mathbb{D}} \quad \text { при } n \rightarrow \infty \text {. }
$$

Выше было замечено, что $\Phi(z)=z \varphi^{\prime}(z) / \varphi(z)$. Следовательно $a_{k}=k \gamma_{k}$, где $\gamma_{k}-$ логарифмические коэффициенты однолистной функции $\varphi$, т.е. коэффициенты Тейлора функции $\ln (\varphi(z) / z)$. Из результатов Смита и Стегенги [6] следует, что существует $\alpha>0$ такое, что

$$
\sum_{k=1}^{\infty} k^{1+\alpha}\left|\gamma_{k}\right|^{2}<\infty
$$

откуда

$$
\sum_{k=1}^{\infty}\left|\gamma_{k}\right|<\infty
$$

и, следовательно,

$$
\sum_{k=1}^{\infty} \frac{\left|a_{k}\right|}{k}<\infty
$$

Следует отметить, что Смит и Стегенга доказали неравенство для коэффициентов гёльдеровых функций (а следовательно, и для квазиконформных) отображений $\varphi$, оценивая интегральные средние от модулей производных с использованием классических неравенств между интегральными средними и коэффициентами, восходящими к Литтлвуду. В силу того, что $\varphi$ отделено от нуля и бесконечности, неравенство (7) останется справедливым, если заменить коэффициенты функции на логарифмические, поскольку интегральные средние от $\left|\varphi^{\prime}\right|$ и $\left|z \varphi^{\prime} / \varphi\right|$ сравнимы.

Из (7) следует, что

$$
\ln \frac{\varphi_{n}(z)}{z}=n \ln \left(1+\sum_{k=1}^{\infty} \frac{a_{k}}{n k+1} z^{k}\right)=n \sum_{k=1}^{\infty} \frac{a_{k}}{n k+1} z^{k}+O\left(\frac{1}{n}\right) .
$$

Несложный анализ показывает, что последнее выражение сходится к

$$
\sum_{k=1}^{\infty} \frac{a_{k}}{k} z^{k}=\ln \frac{\varphi(z)}{z}
$$

при $n \rightarrow \infty$ равномерно в $\overline{\mathbb{D}}$, что доказывает (6), и как следствие $(5)$, потому, что функция $\ln (\varphi(z) / z)$ отделена от нуля и бесконечности, так что второе слагаемое последнего выражения в (4) равномерно стремится к нулю при $n \rightarrow \infty$.

Применяя лемму Шварца к (5), получаем, что

$$
\left\|\ln \varphi_{n}^{\prime}-\ln \varphi^{\prime}\right\|_{\mathbb{B}} \rightarrow 0 \quad \text { при } \quad n \rightarrow \infty,
$$


где $\|h\|_{\mathbb{B}}=\sup _{|z|<1}\left(1-|z|^{2}\right)\left|h^{\prime}(z)\right|-$ норма Блоха. Это значит, что для любого $\varepsilon>0$ существует натуральное $N$ такое, что для всех $n \geqslant N$ и $z \in \mathbb{D}$ выполнено неравенство

$$
\left|\frac{\varphi_{n}^{\prime \prime}(z)}{\varphi_{n}^{\prime}(z)}-\frac{\varphi^{\prime \prime}(z)}{\varphi^{\prime}(z)}\right| \leqslant \frac{\varepsilon}{1-|z|^{2}} .
$$

Полагая $\psi_{n}=\varphi_{n} \circ \varphi^{-1}$ и применяя (8), получаем (3), что доказывает однолистность функции $\psi_{n}$, а следовательно, и $\varphi_{n}$ в $\mathbb{D}$ при достаточно больших $n$. Теорема 1 доказана.

В дальнейшем нам потребуется

ОПРЕДЕЛЕНИЕ 1 . Пусть $r$ - некоторое положительное число, $E$ - некоторое подмножество $\overline{\mathbb{C}}, \mathbb{D}_{r}=\{\lambda:|\lambda|<r\}$. Голоморфным движением $E$ называется отображение $h: \mathbb{D}_{r} \times E \rightarrow \overline{\mathbb{C}}$, удовлетворяющее условиям

(a) для любого фиксированного $z \in E$ функция $h(\lambda, z)$ голоморфна в круге $\mathbb{D}_{r}$;

(b) для любого фиксированного $\lambda \in \mathbb{D}_{r}$ функция $h(\lambda, z)$ инъективна в $E$;

(c) $h(0, z)=z$ для всех $z \in E$.

Определение 1 дано в работе Мане, Сада и Сулливана [7] для $r=1$. Параметр $r>0$ введен нами для удобства.

Пусть функция $\Phi(\lambda, z)$ голоморфна в $\mathbb{D}_{r} \times \mathbb{D}$ и $\Phi(0, z)=1$ для всех $z \in \mathbb{D}$. Положим

$$
f_{n}(\lambda, z)=\int_{0}^{z} \Phi\left(\lambda, t^{n}\right) d t
$$

Выше нами была сформулирована основная проблема, которую мы теперь уточним (в связи с появлением дополнительно параметра $\lambda$ ): найти максимальное $\lambda(n)$ такое, что функции (9) однолистны в $\mathbb{D}$ при любых значениях параметра $\lambda$, удовлетворяющего неравенству $|\lambda| \leqslant \lambda(n)$.

Положим

$$
\begin{gathered}
\varphi(\lambda, z)=z \exp \int_{0}^{z} \frac{\Phi(\lambda, t)-1}{t} d t, \\
\lambda_{\min }=\inf \{|\lambda|: \varphi(\lambda, z) \text { не однолистна в } \mathbb{D}\}, \\
\lambda_{\max }=\max \{|\lambda|: \varphi(\lambda, z) \text { однолистна в } \mathbb{D}\} .
\end{gathered}
$$

Следующая теорема дает "асимптотическое решение" поставленной проблемы.

Теорема 2. Пусть $\lambda$-некоторое комплексное число. Если существует натуралъное $N$ такое, что функции $f_{n}(\lambda, z)$ однолистны (по переменному $z$ ) в $\mathbb{D}$ для всех $n \geqslant N$, mo $|\lambda| \leqslant \lambda_{\max }$.

Обратно, если $|\lambda|<\lambda_{\min }$, то найдется натуральное число $N$ такое, что функиии $f_{n}(\lambda, z)$ однолистнъ в $\mathbb{D}$ при $n \geqslant N$.

ДокАЗАтЕЛьство. Предположим, что существует натуральное $N$ такое, что функции $f_{n}(\lambda, z)$ однолистны (по переменной $z$ ) в $\mathbb{D}$ для всех $n \geqslant N$. Тогда часть а) теоремы 1 влечет, что функции (10) однолистны (по переменной $z$ ) в круге $\mathbb{D}$. Следовательно, $|\lambda| \leqslant \lambda_{\max }$, что доказывает первую часть теоремы 2.

Докажем теперь вторую часть теоремы 2. Для этого предположим, что $|\lambda|<\lambda_{\min }$. Нетрудно видеть, что функция $\varphi(\lambda, z)$ является голоморфным движением на $\mathbb{D}_{r} \times E$, 
где $r=\lambda_{\min }$ и $E=\mathbb{D}$. В силу $\lambda$-леммы Мане, Сада и Сулливана [7], функция $\varphi(\lambda, z)$ является квазиконформным отображением круга $\mathbb{D}$ для любого фиксированного $\lambda \in \mathbb{D}_{\lambda_{\min }}$. Теперь из части b) теоремы 1 следует, что для любого $\lambda \in \mathbb{D}_{\lambda_{\min }}$ найдется натуральное $N$ такое, что функции (9) однолистны в круге $\mathbb{D}$. Теорема 2 доказана.

Заметим, что, используя теорему 2 , легко проверить, что функция $f_{n}(\lambda, z)$ является голоморфным движением на множестве $\mathbb{D}_{\lambda_{\min }} \times \mathbb{D}$ для достаточно большого $n$. Снова применяя $\lambda$-лемму Мане, Сада и Сулливана заключаем, что если $|\lambda|<\lambda_{\min }$, то найдется натуральное $N$ такое, что функции $f_{n}(\lambda, z)$ являются квазиконформными отображениями $\overline{\mathbb{D}}$ для любых $n \geqslant N$.

Чтобы определить $\lambda_{\min }$ и $\lambda_{\max }$ нужно исследовать однолистность однопараметрического семейства функций (10), для чего нужно задать $\Phi$. Во многих случаях $\lambda_{\min }=\lambda_{\max }$. Например, в исходной задаче $\Phi(\lambda, t)=\exp (\lambda t)$,

$$
\varphi(\lambda, z)=z \exp \int_{0}^{z} \frac{e^{\lambda t}-1}{t} d t .
$$

Ясно, что $\lambda_{\min }=\lambda_{\max }$. В работе [8] показано, что функция $\varphi(\lambda, z)$ однолистна в $\mathbb{D}$ для любого $\lambda$ такого, что $|\lambda| \leqslant \lambda_{\min }$, где

$$
\lambda_{\min }=\frac{\pi}{2 \sin \theta} .
$$

Здесь $\theta-$ единственный корень уравнения

$$
\theta=\int_{0}^{\pi / 2} \frac{\sin x}{x} \exp (-x \operatorname{ctg} \theta) d x
$$

на интервале $(0, \pi / 2)$. Следовательно, $\lambda_{\min }=\lambda_{\max }=1.7646 \ldots$.

Теорема 1, доказанная выше, позволяет сформулировать такую гипотезу.

ГиПотезА. Пусть $\Phi(z)$ голоморфна в $\mathbb{D} u \Phi(0)=1$. Тогда функиия

$$
\varphi(z)=z \exp \int_{0}^{z} \frac{\Phi(t)-1}{t} d t
$$

однолистна в круге $\mathbb{D}$ тогда и только тогда, когда существует натуралъное $N$ такое, что функиии

$$
f_{n}(z)=\int_{0}^{z} \Phi\left(t^{n}\right) d t
$$

однолистны в круге $\mathbb{D}$ при всех $n \geqslant N$.

Другими словами, эта гипотеза утверждает, что квазиконформность в теореме 1 может быть ослаблена до однолистности.

Интересно отметить, что наша гипотеза следует из гипотезы, сформулированной Даникасом и Рушевеем в [9]. Они предположили, что если голоморфная функция $f$ однолистна в круге $\mathbb{D}$, то функция

$$
g(z)=\int_{0}^{z} t \frac{f^{\prime}(t)}{f(t)} d t
$$


также однолистна в этом круге. Применяя преобразование Фабера $\sqrt[n]{f\left(z^{n}\right)}$ к этим функциям, заключаем, что функция

$$
g(z)=\int_{0}^{z} t^{n} \frac{f^{\prime}\left(t^{n}\right)}{f\left(t^{n}\right)} d t
$$

будет однолистной для всех $n$.

Предполагая справедливость гипотезы Даникаса-Рушевея, применим ее к функции

$$
f(z)=\int_{0}^{z} t^{n} \frac{\varphi^{\prime}\left(t^{n}\right)}{\varphi\left(t^{n}\right)} d t=\int_{0}^{z} \Phi\left(t^{n}\right) d t .
$$

Это дает однолистность $f_{n}(z)$ в круге $\mathbb{D}$ для всех натуральных $n$, в то время как наша гипотеза утверждает, что эти функции однолистны для достаточно больших значений $n$. Таким образом, наша гипотеза немного слабее гипотезы Даникаса-Рушевея. Преимущество нашей гипотезы заключается в том, что утверждение является симметричным, в то время как обратное утверждение к гипотезе Даникаса-Рушевея является неверным.

Нетрудно показать, что если функция $f_{n}(z)$ почти выпукла в круге $\mathbb{D}$ для некоторого натурального $n$, то $f_{1}(z)$ однолистна в круге $\mathbb{D}$. Мы предполагаем, что данный факт справедлив во всем классе однолистных функций. Гипотеза Даникаса-Рушевея следует из этого предположения. Для доказательства этого утверждения можно считать, что функция

$$
\varphi(z)=z \exp \int_{0}^{z} \frac{\Phi(t)-1}{t} d t
$$

отображает круг $\mathbb{D}$ на односвязную область с квазиконформной границей, что позволяет использовать часть b) теоремы 1 . Таким образом, $f_{n}(z)$ однолистна в круге $\mathbb{D}$ для некоторого натурального $n$. В силу нашего предположения $f_{1}(z)$ также однолистна в этом круге. Для вывода отсюда гипотезы Даникаса-Рушевея остается только заметить, что $f_{1}^{\prime}(z)=z \varphi^{\prime}(z) / \varphi^{\prime}(z)$.

Часть b) теоремы 1 может быть слегка усилена, если вместо достаточного условия однолистности (3) использовать другое условие. Пусть $\Omega$ - односвязная область с квазиконформной границей. Тогда найдется $\varepsilon>0$ такое, что если голоморфная функция $\psi$ удовлетворяет в $\Omega$ условию

$$
\left|\arg \psi^{\prime}\right|<\varepsilon,
$$

то $\psi$ однолистна в $\Omega$. Достаточное условие однолистности (11) может быть получено из условия однолистности (3). Однако, области с квазиконформными границами являются естественным классом применения условия (3), т.е. если квазиконформности нет, то для любого $\varepsilon>0$ найдется неоднолистная в области $\Omega$ функция, удовлетворяющая условию (3). Достаточное условие однолистности (11) является более гибким, т.е. может применяться к областям, чьи границы не обязательно квазиконформны. Авхадиев [10] показал, что во многих случаях условие квазиконформности может быть заменено условием отсутствия внешних нулевых углов границы области.

\section{СПИСОК ЦИТИРОВАННОЙ ЛИТЕРАТУРЫ}

[1] J. Becker, "Löwnersche Differentialgleichung und quasikonform fortsetzbare schlichte Funktionen", J. Reine Angew. Math., 255 (1972), 23-43. 
[2] P. L. Duren, Univalent Functions, Grundlehren Math. Wiss., 259, Springer-Verlag, New York, 1983.

[3] В.Н.Гайдук, "Об однолистности решений обратных краевых задач”, Тр. сем. по краев. задачам, 9, Изд-во Казанского ун-та, Казань, 1972, 39-48.

[4] Г. М. Голузин, Геометрическал теория функиий комплексного переменного, Гостехиздат, М.-Л., 1952.

[5] O. Martio, J. Sarvas, "Injectivity theorems in plane and space", Ann. Acad. Sci. Fenn. Ser. A I Math., 4:2 (1979), 383-401.

[6] W. Smith, D. A. Stegenga, "Exponential integrability of the quasi-hyperbolic metric on Hölder domains", Ann. Acad. Sci. Fenn. Ser. A I Math., 16:2 (1991), 345-360.

[7] R. Mañé, P. Sad, D. Sullivan, "On the dynamics of rational maps", Ann. Sci. École Norm. Sup. (4), 16:2 (1983), 193-217.

[8] I. Kayumov, "Lower estimate for the integral means spectrum for $p=-1$ ", Proc. Amer. Math. Soc., 130:4 (2002), 1005-1007.

[9] N. Danikas, S. Ruscheweyh, "Semi-convex hulls of analytic functions in the unit disk", Analysis (Munich), 19:4 (1999), 309-318.

[10] Ф.Г. Авхадиев, Конформные отображения и краевые задачи, Монографии по математике, 2, Казанский фонд "Математика", Казань, 1996.

И. Р. Каюмов

Казанский государственный университет

E-mail: ikayumov@mail.ru
Поступило

19.02.2010

Исправленный вариант

22.04 .2010 